\title{
CHARACTERIZATIONS OF FIXED POINTS OF NONEXPANSIVE MAPPINGS
}

TOMONARI SUZUKI

Received 14 September 2004 and in revised form 24 March 2005

Using the notion of Banach limits, we discuss the characterization of fixed points of nonexpansive mappings in Banach spaces. Indeed, we prove that the two sets of fixed points of a nonexpansive mapping and some mapping generated by a Banach limit coincide. In our discussion, we may not assume the strict convexity of the Banach space.

\section{Introduction}

Let $C$ be a closed convex subset of a Banach space $E$. A mapping $T$ on $C$ is called a nonexpansive mapping if $\|T x-T y\| \leq\|x-y\|$ for all $x, y \in C$. We denote by $F(T)$ the set of fixed points of $T$. Kirk [17] proved that $F(T)$ is nonempty in the case that $C$ is weakly compact and has normal structure. See also $[2,3,5,6,11]$ and others.

Convergence theorems to fixed points are also proved by many authors; see $[1,7,8$, $9,10,13,15,18,23,30]$ and others. Very recently, the author proved the convergence theorems for two nonexpansive mappings without the assumption of the strict convexity of the Banach space. To prove this, the author proved the following theorem, which plays an extremely important role in [26].

Theorem 1.1 (see [26]). Let $C$ be a compact convex subset of a Banach space $E$ and let $T$ be a nonexpansive mapping on $C$. Then $z \in C$ is a fixed point of $T$ if and only if

$$
\liminf _{n \rightarrow \infty}\left\|\frac{1}{n} \sum_{i=1}^{n} T^{i} z-z\right\|=0
$$

holds.

The author also proved the following theorem. Using it, we give one nonexpansive retraction onto the set of all fixed points.

Theorem 1.2 (see [27]). Let $E$ be a Banach space with the Opial property and let $C$ be a weakly compact convex subset of $E$. Let $T$ be a nonexpansive mapping on $C$. Put

$$
M(n, x)=\frac{1}{n} \sum_{i=1}^{n} T^{i} x
$$


for $n \in \mathbb{N}$ and $x \in C$. Then for $z \in C$, the following are equivalent:

(i) $z$ is a fixed point of $T$;

(ii) $\{M(n, z)\}$ converges weakly to $z$;

(iii) there exists a subnet $\left\{M\left(\nu_{\beta}, z\right): \beta \in D\right\}$ of a sequence $\{M(n, z)\}$ in $C$ converging weakly to $z$.

In this paper, using the notion of Banach limits, we generalize Theorems 1.1 and 1.2. We remark that the proofs of our results are simpler than the proofs of Theorems 1.1 and 1.2. In our discussion, we may not assume the strict convexity of the Banach space.

\section{Preliminaries}

Throughout this paper, we denote by $\mathbb{N}$ the set of all positive integers and by $\mathbb{R}$ the set of all real numbers. For a subset $A$ of $\mathbb{N}$, we define a function $I_{A}$ from $\mathbb{N}$ into $\mathbb{R}$ by

$$
I_{A}(n)= \begin{cases}1 & \text { if } n \in A, \\ 0 & \text { if } n \notin A .\end{cases}
$$

Let $E$ be a Banach space. We denote by $E^{*}$ the dual of $E$. We recall that $E$ is said to have the Opial property [21] if for each weakly convergent sequence $\left\{x_{n}\right\}$ in $E$ with weak $\operatorname{limit} x_{0}, \liminf _{n}\left\|x_{n}-x_{0}\right\|<\liminf _{n}\left\|x_{n}-x\right\|$ for all $x \in E$ with $x \neq x_{0}$. All Hilbert spaces, all finite-dimensional Banach spaces, and $\ell^{p}(1 \leq p<\infty)$ have the Opial property. A Banach space with a duality mapping which is weakly sequentially continuous also has the Opial property; see [12]. We know that every separable Banach space can be equivalently renormed so that it has the Opial property; see [31]. Gossez and Lami Dozo [12] prove that every weakly compact convex subset of a Banach space with the Opial property has normal structure. See also $[19,20,22,25]$ and others.

We denote by $\ell^{\infty}$ the Banach space consisting of all bounded functions from $\mathbb{N}$ into $\mathbb{R}$ (i.e., all bounded real sequences) with supremum norm. We recall that $\mu \in\left(\ell^{\infty}\right)^{*}$ is called a mean if $\|\mu\|=\mu\left(I_{\mathbb{N}}\right)=1$. It is equivalent to

$$
\inf _{n \in \mathbb{N}} a(n) \leq \mu(a) \leq \sup _{n \in \mathbb{N}} a(n)
$$

for all $a \in \ell^{\infty}$. We also know that if $a(n) \leq b(n)$ for all $n \in \mathbb{N}$, then $\mu(a) \leq \mu(b)$ holds. Sometimes, we denote by $\mu_{n}(a(n))$ the value $\mu(a) . \mu \in\left(\ell^{\infty}\right)^{*}$ is called a Banach limit if the following hold:

(i) $\mu$ is a mean;

(ii) $\mu(a)=\mu_{n}(a(n+1))$ for all $a \in \ell^{\infty}$. That is, putting $b(n)=a(n+1)$ for $n \in \mathbb{N}$, we have $\mu(a)=\mu(b)$.

It is obvious that

$$
\mu(a)=\mu_{n}(a(n+k))
$$


for a Banach limit $\mu, a \in \ell^{\infty}$, and $k \in \mathbb{N}$. We know that Banach limits exist; see [4]. We also know that

$$
\liminf _{n \rightarrow \infty} a(n) \leq \mu(a) \leq \limsup _{n \rightarrow \infty} a(n)
$$

for all $a \in \ell^{\infty}$.

Let $T$ be a nonexpansive mapping on a weakly compact convex subset $C$ of a Banach space $E$. Let $\mu$ be a Banach limit. Then we know that for $x \in C$, there exists a unique element $x_{0}$ of $C$ satisfying

$$
\mu_{n}\left(f\left(T^{n} x\right)\right)=f\left(x_{0}\right)
$$

for all $f \in E^{*}$; see $[14,19]$. Following Rodé [24], we denote such $x_{0}$ by $T_{\mu} x$. We also know that $T_{\mu}$ is a nonexpansive mapping on $C$.

We now prove the following lemmas, which are used in Section 3.

Lemma 2.1. Let $a, b \in \ell^{\infty}$ and let $\mu$ be a Banach limit. Then the following hold.

(i) If there exists $n_{0} \in \mathbb{N}$ such that $a(n) \leq b(n)$ for all $n \geq n_{0}$, then $\mu(a) \leq \mu(b)$ holds.

(ii) If there exists $n_{0} \in \mathbb{N}$ such that $a(n)=b(n)$ for all $n \geq n_{0}$, then $\mu(a)=\mu(b)$ holds.

Proof. We first show (i). We note that $a\left(n_{0}+n\right) \leq b\left(n_{0}+n\right)$ for all $n \in \mathbb{N}$. Since $\mu$ is a Banach limit, we have

$$
\mu_{n}(a(n))=\mu_{n}\left(a\left(n_{0}+n\right)\right) \leq \mu_{n}\left(b\left(n_{0}+n\right)\right)=\mu_{n}(b(n)) .
$$

It is obvious that (ii) follows from (i). This completes the proof.

Lemma 2.2. Let $A_{1}, A_{2}, A_{3}, \ldots, A_{k}$ be subsets of $\mathbb{N}$ and let $\mu$ be a Banach limit. Put

$$
A=\bigcap_{j=1}^{k} A_{j}, \quad \alpha=\sum_{j=1}^{k} \mu\left(I_{A_{j}}\right)-k+1 .
$$

Suppose that $\alpha>0$. Then,

$$
\mu\left(I_{A}\right) \geq \alpha
$$

holds and

$$
\left\{n \in \mathbb{N}: n \geq n_{0}\right\} \cap A \neq \varnothing
$$

holds for all $n_{0} \in \mathbb{N}$.

Proof. It is obvious that $n \in A$ if and only if

$$
\sum_{j=1}^{k} I_{A_{j}}(n)=k
$$


1726 Characterizations of fixed points

and $n \in \mathbb{N} \backslash A$ if and only if

$$
\sum_{j=1}^{k} I_{A_{j}}(n) \leq k-1 .
$$

Therefore we obtain

$$
I_{A}(n) \geq \sum_{j=1}^{k} I_{A_{j}}(n)-k+1
$$

for all $n \in \mathbb{N}$. Hence,

$$
\begin{aligned}
\mu\left(I_{A}\right) & \geq \mu_{n}\left(\sum_{j=1}^{k} I_{A_{j}}(n)-k+1\right) \\
& =\sum_{j=1}^{k} \mu\left(I_{A_{j}}\right)-k+1 \\
& =\alpha>0 .
\end{aligned}
$$

We suppose that $\left\{n \in \mathbb{N}: n \geq n_{0}\right\} \cap A=\varnothing$ for some $n_{0} \in \mathbb{N}$. Then $I_{A}(n)=0$ for $n \geq n_{0}$. So, from Lemma 2.1, we obtain

$$
0<\alpha \leq \mu\left(I_{A}\right)=\mu(0)=0 .
$$

This is a contradiction. This completes the proof.

\section{Main results}

In this section, we prove our main results.

We first prove the following theorem, which plays an important role in this paper.

Theorem 3.1. Let $E$ be a Banach space and let $C$ be a weakly compact convex subset of $E$. Let $T$ be a nonexpansive mapping on $C$. Let $\mu$ be a Banach limit. Suppose that $T_{\mu} z=z$ for some $z \in C$. Then there exist sequences $\left\{p_{n}\right\}$ in $\mathbb{N}$ and $\left\{f_{n}\right\}$ in $E^{*}$ such that

$$
\begin{gathered}
p_{n+1}>p_{n}, \\
\left\|T^{p_{n}} z-z\right\| \geq \lambda-\frac{1}{3^{n+1}}, \\
f_{\ell}\left(T^{p_{n}} z-z\right) \leq \frac{2^{\ell+1}}{3^{\ell+1}} \quad \text { for } \ell=1,2, \ldots, n-1, \\
\left\|f_{n}\right\|=1, \quad f_{n}\left(T^{p_{n}} z-z\right)=\left\|T^{p_{n}} z-z\right\|
\end{gathered}
$$

for all $n \in \mathbb{N}$, where

$$
\lambda=\limsup _{n \rightarrow \infty}\left\|T^{n} z-z\right\|
$$


Before proving this theorem, we need some preliminaries. In the following lemmas and the proof of Theorem 3.1, we put

$$
A(f, \varepsilon)=\left\{n \in \mathbb{N}: f\left(T^{n} z-z\right) \leq \varepsilon\right\}
$$

for $f \in E^{*}$ and $\varepsilon>0$, and

$$
B(\varepsilon)=\left\{n \in \mathbb{N}:\left\|T^{n} z-z\right\| \geq \lambda-\varepsilon\right\}
$$

for $\varepsilon>0$.

Lemma 3.2. For every $n \in \mathbb{N}$,

$$
\left\|T^{n} z-z\right\| \leq \lambda
$$

holds.

Proof. Since $\mu$ is a Banach limit, we have $\mu_{n}\left(\left\|T^{n} z-z\right\|\right) \leq \lambda$. Fix $m \in \mathbb{N}$. By the HahnBanach theorem, there exists $f \in E^{*}$ such that

$$
\|f\|=1, \quad f\left(T^{m} z-z\right)=\left\|T^{m} z-z\right\| .
$$

For $n \in \mathbb{N}$, we have

$$
\begin{aligned}
\left\|T^{m} z-z\right\| & =f\left(T^{m} z-z\right) \\
& =f\left(T^{m} z-T^{m+n} z\right)+f\left(T^{m+n} z-z\right) \\
& \leq\|f\|\left\|T^{m} z-T^{m+n} z\right\|+f\left(T^{m+n} z-z\right) \\
& =\left\|T^{m} z-T^{m+n} z\right\|+f\left(T^{m+n} z-z\right) \\
& \leq\left\|T^{n} z-z\right\|+f\left(T^{m+n} z-z\right) .
\end{aligned}
$$

Hence

$$
\begin{aligned}
\left\|T^{m} z-z\right\| & =\mu_{n}\left(\left\|T^{m} z-z\right\|\right) \\
& \leq \mu_{n}\left(\left\|T^{n} z-z\right\|+f\left(T^{m+n} z-z\right)\right) \\
& =\mu_{n}\left(\left\|T^{n} z-z\right\|\right)+\mu_{n}\left(f\left(T^{m+n} z\right)\right)-f(z) \\
& =\mu_{n}\left(\left\|T^{n} z-z\right\|\right)+\mu_{n}\left(f\left(T^{n} z\right)\right)-f(z) \\
& =\mu_{n}\left(\left\|T^{n} z-z\right\|\right)+f\left(T_{\mu} z\right)-f(z) \\
& =\mu_{n}\left(\left\|T^{n} z-z\right\|\right) \\
& \leq \lambda .
\end{aligned}
$$

This completes the proof. 
1728 Characterizations of fixed points

Lemma 3.3. Suppose that $m \in \mathbb{N}, f \in E^{*}$, and $\delta>0$ satisfy that

$$
\|f\|=1, \quad f\left(T^{m} z-z\right)=\left\|T^{m} z-z\right\| \geq \lambda-\delta .
$$

Then

$$
\mu\left(I_{A(f, \varepsilon)}\right) \geq \frac{\varepsilon}{\varepsilon+\delta}
$$

holds for all $\varepsilon>0$.

Proof. For $n>m$, by Lemma 3.2, we have

$$
\begin{aligned}
f\left(T^{n} z-z\right) & =f\left(T^{n} z-T^{m} z\right)+f\left(T^{m} z-z\right) \\
& \geq-\|f\|\left\|T^{n} z-T^{m} z\right\|+f\left(T^{m} z-z\right) \\
& =-\left\|T^{n} z-T^{m} z\right\|+\left\|T^{m} z-z\right\| \\
& \geq-\left\|T^{n-m} z-z\right\|+\left\|T^{m} z-z\right\| \\
& \geq-\lambda+\lambda-\delta=-\delta .
\end{aligned}
$$

On the other hand, by the definition of $A(f, \varepsilon)$,

$$
f\left(T^{n} z-z\right)>\varepsilon
$$

for all $n \in \mathbb{N} \backslash A(f, \varepsilon)$. Therefore, for $n \in \mathbb{N}$ with $n>m$, we have

$$
\begin{aligned}
f\left(T^{n} z-z\right) & \geq-\delta I_{A(f, \varepsilon)}(n)+\varepsilon I_{\mathbb{N} \backslash A(f, \varepsilon)}(n) \\
& =-\delta I_{A(f, \varepsilon)}(n)+\varepsilon I_{\mathbb{N} \backslash A(f, \varepsilon)}(n)+(\varepsilon-\varepsilon) I_{A(f, \varepsilon)}(n) \\
& =-(\delta+\varepsilon) I_{A(f, \varepsilon)}(n)+\varepsilon I_{\mathbb{N}}(n) \\
& =-(\delta+\varepsilon) I_{A(f, \varepsilon)}(n)+\varepsilon .
\end{aligned}
$$

By Lemma 2.1, we have

$$
\begin{aligned}
0 & =f\left(T_{\mu} z\right)-f(z) \\
& =\mu_{n}\left(f\left(T^{n} z\right)\right)-f(z) \\
& =\mu_{n}\left(f\left(T^{n} z-z\right)\right) \\
& \geq \mu_{n}\left(-(\delta+\varepsilon) I_{A(f, \varepsilon)}(n)+\varepsilon\right) \\
& =-(\delta+\varepsilon) \mu\left(I_{A(f, \varepsilon)}\right)+\varepsilon .
\end{aligned}
$$

Hence, we obtain

$$
\mu\left(I_{A(f, \varepsilon)}\right) \geq \frac{\varepsilon}{\varepsilon+\delta}
$$

This completes the proof. 
LEMma 3.4. $\mu\left(I_{B(\varepsilon)}\right)=1$ holds for all $\varepsilon>0$.

Proof. We fix $\varepsilon>0$ and $\eta \in \mathbb{R}$ with $1 / 2<\eta<1$ and put

$$
\delta=\frac{\varepsilon(1-\eta)}{2 \eta} .
$$

We note that $0<\delta<\varepsilon / 2$. By the definition of $\lambda$, there exists $m \in \mathbb{N}$ such that

$$
\left\|T^{m} z-z\right\| \geq \lambda-\delta
$$

Fix $f \in E^{*}$ with

$$
\|f\|=1, \quad f\left(T^{m} z-z\right)=\left\|T^{m} z-z\right\| .
$$

So, using Lemma 3.3, we have

$$
\mu\left(I_{A(f, \varepsilon / 2)}\right) \geq \frac{\varepsilon / 2}{\varepsilon / 2+\delta}=\eta .
$$

For $n \in \mathbb{N}$ with $m+n \in A(f, \varepsilon / 2)$, we have

$$
\begin{aligned}
\left\|T^{n} z-z\right\| & \geq\left\|T^{m} z-T^{m+n} z\right\| \\
& \geq f\left(T^{m} z-T^{m+n} z\right) \\
& =f\left(T^{m} z-z\right)+f\left(z-T^{m+n} z\right) \\
& =\left\|T^{m} z-z\right\|+f\left(z-T^{m+n} z\right) \\
& \geq \lambda-\delta-\frac{\varepsilon}{2} \\
& \geq \lambda-\varepsilon,
\end{aligned}
$$

and hence $n \in B(\varepsilon)$. Therefore

$$
I_{B(\varepsilon)}(n) \geq I_{A(f, \varepsilon / 2)}(m+n)
$$

for all $n \in \mathbb{N}$. So we obtain

$$
\begin{aligned}
\mu\left(I_{B(\varepsilon)}\right) & \geq \mu_{n}\left(I_{A(f, \varepsilon / 2)}(m+n)\right) \\
& =\mu_{n}\left(I_{A(f, \varepsilon / 2)}(n)\right) \\
& \geq \eta .
\end{aligned}
$$

Since $\eta$ is arbitrary, we obtain the desired result.

Proof of Theorem 3.1. By the definition of $\lambda$, there exists $p_{1} \in \mathbb{N}$ such that

$$
\left\|T^{p_{1}} z-z\right\| \geq \lambda-\frac{1}{3^{2}}
$$

Fix $f_{1} \in E^{*}$ with

$$
\left\|f_{1}\right\|=1, \quad f_{1}\left(T^{p_{1}} z-z\right)=\left\|T^{p_{1}} z-z\right\| .
$$


1730 Characterizations of fixed points

By Lemma 3.3, we have

$$
\mu\left(I_{A\left(f_{1},(2 / 3)^{2}\right)}\right) \geq \frac{2^{2}}{2^{2}+1} .
$$

We now define inductively sequences $\left\{p_{n}\right\}$ in $\mathbb{N}$ and $\left\{f_{n}\right\}$ in $E^{*}$. Suppose that $p_{k} \in \mathbb{N}$ and $f_{k} \in E^{*}$ are known. Since

$$
\begin{aligned}
& \mu\left(I_{B\left(1 / 3^{k+2}\right)}\right)+\sum_{\ell=1}^{k} \mu\left(I_{A\left(f_{\ell},(2 / 3)^{\ell+1}\right)}\right)-k \\
& \geq 1+\sum_{\ell=1}^{k} \frac{2^{\ell+1}}{2^{\ell+1}+1}-k \\
& \geq 1+\sum_{\ell=1}^{k} \frac{2^{\ell+1}-1}{2^{\ell+1}}-k=1+\sum_{\ell=1}^{k} \frac{-1}{2^{\ell+1}} \\
& >\frac{1}{2}>0,
\end{aligned}
$$

we have

$$
\left\{m \in \mathbb{N}: m \geq p_{k}+1\right\} \cap B\left(\frac{1}{3^{k+2}}\right) \cap \bigcap_{\ell=1}^{k} A\left(f_{\ell},\left(\frac{2}{3}\right)^{\ell+1}\right) \neq \varnothing
$$

by Lemma 2.2. So we can choose $p_{k+1} \in \mathbb{N}$ such that $p_{k+1}>p_{k}$,

$$
\left\|T^{p_{k+1}} z-z\right\| \geq \lambda-\frac{1}{3^{k+2}}, \quad f_{\ell}\left(T^{p_{k+1}} z-z\right) \leq \frac{2^{\ell+1}}{3^{\ell+1}}
$$

for $\ell=1,2, \ldots, k$. Fix $f_{k+1} \in E^{*}$ with

$$
\left\|f_{k+1}\right\|=1, \quad f_{k+1}\left(T^{p_{k+1}} z-z\right)=\left\|T^{p_{k+1}} z-z\right\| .
$$

Note that

$$
\mu\left(I_{A\left(f_{k+1},(2 / 3)^{k+2}\right)}\right) \geq \frac{2^{k+2}}{2^{k+2}+1}
$$

by Lemma 3.3. Hence we have defined $\left\{p_{n}\right\}$ and $\left\{f_{n}\right\}$.

Now, we prove our main results.

Theorem 3.5. Let $C$ be a weakly compact convex subset of a Banach space $E$ with the Opial property. Let $T$ be a nonexpansive mapping on $C$. Let $\mu$ be a Banach limit. Then $z \in C$ is a fixed point of $T$ if and only if $T_{\mu} z=z$. 
Proof. We first assume that $z \in C$ is a fixed point of $T$. Then, we have

$$
f\left(T_{\mu} z\right)=\mu_{n}\left(f\left(T^{n} z\right)\right)=\mu_{n}(f(z))=f(z)
$$

for all $f \in E^{*}$, and hence $T_{\mu} z=z$. Conversely, we assume that $T_{\mu} z=z$. By Theorem 3.1, there exist sequences $\left\{p_{n}\right\}$ in $\mathbb{N}$ and $\left\{f_{n}\right\}$ in $E^{*}$ satisfying the conclusion of Theorem 3.1. We put $\lambda=\limsup _{n}\left\|T^{n} z-z\right\|$. Since $C$ is weakly compact, there exists a subsequence $\left\{p_{n_{k}}\right\}$ of $\left\{p_{n}\right\}$ such that $\left\{T^{p_{n_{k}}} z\right\}$ converges weakly to some point $u \in C$. If $n_{k}>\ell$, then

$$
f_{\ell}\left(T^{p_{n_{k}}} z-z\right) \leq \frac{2^{\ell+1}}{3^{\ell+1}}
$$

So we obtain

$$
f_{\ell}(u-z) \leq \frac{2^{\ell+1}}{3^{\ell+1}}
$$

for all $\ell \in \mathbb{N}$. Since

$$
\begin{aligned}
\left\|T^{p_{\ell}} z-u\right\| & =\left\|f_{\ell}\right\|\left\|T^{p_{\ell}} z-u\right\| \\
& \geq f_{\ell}\left(T^{p_{\ell}} z-u\right) \\
& =f_{\ell}\left(T^{p_{\ell}} z-z\right)+f_{\ell}(z-u) \\
& =\left\|T^{p_{\ell}} z-z\right\|+f_{\ell}(z-u) \\
& \geq \lambda-\frac{1}{3^{\ell+1}}-\frac{2^{\ell+1}}{3^{\ell+1}}
\end{aligned}
$$

for $\ell \in \mathbb{N}$, we have

$$
\liminf _{\ell \rightarrow \infty}\left\|T^{p_{\ell}} z-u\right\| \geq \lambda
$$

and hence

$$
\begin{aligned}
\liminf _{k \rightarrow \infty}\left\|T^{p_{n_{k}}} z-z\right\| & \leq \lambda \leq \liminf _{\ell \rightarrow \infty}\left\|T^{p_{\ell}} z-u\right\| \\
& \leq \liminf _{k \rightarrow \infty}\left\|T^{p_{n_{k}}} z-u\right\| .
\end{aligned}
$$

By the Opial property of $E$, we obtain $z=u$. We also have

$$
\liminf _{k \rightarrow \infty}\left\|T^{p_{n_{k}}} z-T z\right\| \leq \liminf _{k \rightarrow \infty}\left\|T^{p_{n_{k}}-1} z-z\right\| \leq \lambda,
$$

and hence $T z=u$. Therefore $T z=z$. This completes the proof. 
Theorem 3.6. Let $C$ be a compact convex subset of a Banach space E. Let $T$ be a nonexpansive mapping on $C$. Let $\mu$ be a Banach limit. Then $z \in C$ is a fixed point of $T$ if and only if $T_{\mu} z=z$.

Proof. From the proof of Theorem 3.5, we know that $T z=z$ implies that $T_{\mu} z=z$. Conversely, we assume that $T_{\mu} z=z$. By Theorem 3.1, there exist sequences $\left\{p_{n}\right\}$ in $\mathbb{N}$ and $\left\{f_{n}\right\}$ in $E^{*}$ satisfying the conclusion of Theorem 3.1. We put $\lambda=\limsup _{n}\left\|T^{n} z-z\right\|$. Since $C$ is compact, there exists a subsequence $\left\{p_{n_{k}}\right\}$ of $\left\{p_{n}\right\}$ such that $\left\{T^{p_{n_{k}}} z\right\}$ converges strongly to some point $u \in C$. As in the proof of Theorem 3.5, we obtain $\liminf _{\ell}\left\|T^{p_{\ell}} z-u\right\| \geq \lambda$. This implies that $\lambda=0$, and hence $\left\{T^{n} z\right.$ converges to $z$. So we have

$$
T z=T\left(\lim _{n \rightarrow \infty} T^{n} z\right)=\lim _{n \rightarrow \infty} T^{n+1} z=z
$$

This completes the proof.

\section{Appendix}

In some sense, Theorems 3.5 and 3.6 are generalizations of Theorems 1.2 and 1.1, respectively. To show this, we use the notion of universal nets. We recall that a net $\left\{y_{\beta}: \beta \in D\right\}$ in a topological space $Y$ is universal if for each subset $A$ of $Y$, there exists $\beta_{0} \in D$ satisfying either of the following:

(i) $y_{\beta} \in A$ for all $\beta \in D$ with $\beta \geq \beta_{0}$; or

(ii) $y_{\beta} \in Y \backslash A$ for all $\beta \in D$ with $\beta \geq \beta_{0}$.

For every net $\left\{y_{\beta}: \beta \in D\right\}$, by the axiom of choice, there exists a universal subnet $\left\{y_{\beta_{\gamma}}\right.$ : $\left.\gamma \in D^{\prime}\right\}$ of $\left\{y_{\beta}: \beta \in D\right\}$. If $f$ is a mapping from $Y$ into a topological space $Z$ and $\left\{y_{\beta}\right.$ : $\beta \in D\}$ is a universal net in $Y$, then $\left\{f\left(y_{\beta}\right): \beta \in D\right\}$ is also a universal net in $Z$. If $Y$ is compact, then a universal net $\left\{y_{\beta}: \beta \in D\right\}$ in $Y$ always converges. See [16] and others for details.

Proposition A.1. Let $\left\{\nu_{\beta}: \beta \in D\right\}$ be a universal subnet of a sequence $\{n: n \in \mathbb{N}\}$ in $\mathbb{N}$. Define a function $\mu$ from $\ell^{\infty}$ into $\mathbb{R}$ by

$$
\mu(a)=\lim _{\beta \in D} \frac{1}{\nu_{\beta}} \sum_{i=1}^{\nu_{\beta}} a(i)
$$

for all $a \in \ell^{\infty}$. Then $\mu$ is a Banach limit.

Proof. We note that $\mu$ is well defined because $\left\{\nu_{\beta}: \beta \in D\right\}$ is universal. It is obvious that $\mu$ is linear. For $a \in \ell^{\infty}$, we have

$$
\mu(a)=\lim _{\beta \in D} \frac{1}{\nu_{\beta}} \sum_{i=1}^{\nu_{\beta}} a(i) \leq \lim _{\beta \in D} \frac{1}{\nu_{\beta}} \sum_{i=1}^{\nu_{\beta}}\|a\|=\lim _{\beta \in D}\|a\|=\|a\| .
$$


Similarly, we obtain $\mu(a) \geq-\|a\|$. Hence $\|\mu\| \leq 1$. Since $\mu\left(I_{\mathbb{N}}\right)=1$, we have $\|\mu\|=\mu\left(I_{\mathbb{N}}\right)=$ 1 , that is, $\mu$ is a mean on $\ell^{\infty}$. We also have

$$
\begin{aligned}
\mu_{n}(a(n+1)) & =\lim _{\beta \in D} \frac{1}{v_{\beta}} \sum_{i=1}^{\nu_{\beta}} a(i+1) \\
& =\lim _{\beta \in D}\left(\frac{1}{\nu_{\beta}} \sum_{i=1}^{\nu_{\beta}} a(i)-\frac{a(1)}{\nu_{\beta}}+\frac{a\left(\nu_{\beta}+1\right)}{\nu_{\beta}}\right) \\
& =\mu(a)
\end{aligned}
$$

for all $a \in \ell^{\infty}$. This completes the proof.

Proposition A.2. Let $C$ be a weakly compact convex subset of a Banach space $E$ and let $T$ be a nonexpansive mapping on $C$. Define $M(\cdot, \cdot)$ as in Theorem 1.2. Take a universal subnet $\left\{\nu_{\beta}: \beta \in D\right\}$ of a sequence $\{n: n \in \mathbb{N}\}$ in $\mathbb{N}$ and define a mapping $U$ on $C$ by

$$
U x=\underset{\beta \in D}{\operatorname{weak}} \lim M\left(v_{\beta}, x\right)
$$

for all $x \in C$. Then there exists a Banach limit $\mu$ satisfying $T_{\mu} x=U x$ for all $x \in C$.

Proof. Define a Banach limit $\mu$ as in Proposition A.1. Then for $x \in C$ and $f \in E^{*}$, we have

$$
\begin{aligned}
f(U x) & =\lim _{\beta \in D} f\left(\frac{1}{\nu_{\beta}} \sum_{i=1}^{\nu_{\beta}} T^{i} x\right) \\
& =\lim _{\beta \in D} \frac{1}{v_{\beta}} \sum_{i=1}^{\nu_{\beta}} f\left(T^{i} x\right) \\
& =\mu_{n}\left(f\left(T^{n} x\right)\right) \\
& =f\left(T_{\mu} x\right) .
\end{aligned}
$$

Since $f$ is arbitrary, we have $U x=T_{\mu} x$ for all $x \in C$. This completes the proof.

Using Proposition A.2, we obtain the following proposition.

Proposition A.3. Let $C$ be a weakly compact convex subset of a Banach space $E$ and let $T$ be a nonexpansive mapping on $C$. Then if $z \in C$ satisfies Theorem 1.2(iii), then there exists a Banach limit $\mu$ satisfying $T_{\mu} z=z$.

Proof. Define $M(\cdot, \cdot)$ as in Theorem 1.2. Take a universal subnet $\left\{\nu_{\beta_{\gamma}}: \gamma \in D^{\prime}\right\}$ of $\left\{\nu_{\beta}\right.$ : $\beta \in D\}$. We note that $\left\{\nu_{\beta_{\gamma}}: \gamma \in D^{\prime}\right\}$ is also a universal subnet of a sequence $\{n: n \in \mathbb{N}\}$ in $\mathbb{N}$. We also note that $\left\{M\left(\nu_{\beta_{\gamma}}, z\right): \gamma \in D^{\prime}\right\}$ converges weakly to $z$ because $\left\{M\left(\nu_{\beta}, z\right): \beta \in D\right\}$ does. Define a mapping $U$ on $C$ by

$$
U x=\underset{\gamma \in D^{\prime}}{\operatorname{weak}} \lim M\left(\nu_{\beta_{\gamma}}, x\right)
$$

for all $x \in C$. It is obvious that $U z=z$. By Proposition A.2, there exists a Banach limit $\mu$ satisfying $U=T_{\mu}$. Then we have $T_{\mu} z=z$. This completes the proof. 
In the case that $E$ is a Hilbert space, or $E$ is a uniformly convex Banach space with a Fréchet differentiable norm, $T_{\mu}$ itself is a nonexpansive retraction from $C$ onto $F(T)$; see Baillon [1] and Bruck [8]. In general, this does not hold. We finally give an example.

Example A.4 (see $[27,28])$. Define a compact convex subset $C$ of $\left(\mathbb{R}^{2},\|\cdot\|_{\infty}\right)$ by

$$
C=\left\{\left(x_{1}, x_{2}\right): 0 \leq x_{2} \leq 1,-x_{2} \leq x_{1} \leq x_{2}\right\} .
$$

Define a nonexpansive mapping $T$ on $C$ by

$$
T\left(x_{1}, x_{2}\right)=\left(-x_{1},\left|x_{1}\right|\right)
$$

for all $\left(x_{1}, x_{2}\right) \in C$. Then, $F(T)=\{(0,0)\}$ and

$$
T_{\mu}\left(x_{1}, x_{2}\right)=\left(0,\left|x_{1}\right|\right)
$$

for $\left(x_{1}, x_{2}\right) \in C$ and a Banach limit $\mu$. That is, $T_{\mu}$ is not a nonexpansive retraction from $C$ onto $F(T)$.

\section{References}

[1] J. B. Baillon, Un théorème de type ergodique pour les contractions non linéaires dans un espace de Hilbert, C. R. Acad. Sci. Paris Sér. A-B 280 (1975), no. 22, A1511-A1514 (French).

[2] _ Quelques aspects de la théorie des points fixes dans les espaces de Banach. II, Séminaire d'Analyse Fonctionnelle (1978-1979), Ècole Polytechnique, Palaiseau, 1979, pp. 1-32, exp. no. 8.

[3] _ Quelques aspects de la théorie des points fixes dans les espaces de Banach. I, Séminaire d'Analyse Fonctionnelle (1978-1979), Ėcole Polytechnique, Palaiseau, 1979, pp. 1-13, exp. no. 7.

[4] S. Banach, Théorie des opérations linéaires, Monografie Mat., PWN, Warszawa, 1932.

[5] F. E. Browder, Fixed-point theorems for noncompact mappings in Hilbert space, Proc. Nat. Acad. Sci. U.S.A. 53 (1965), 1272-1276.

[6] Nonexpansive nonlinear operators in a Banach space, Proc. Nat. Acad. Sci. U.S.A. 54 (1965), 1041-1044.

[7] Convergence of approximants to fixed points of nonexpansive non-linear mappings in Banach spaces, Arch. Rational Mech. Anal. 24 (1967), 82-90.

[8] R. E. Bruck, A simple proof of the mean ergodic theorem for nonlinear contractions in Banach spaces, Israel J. Math. 32 (1979), no. 2-3, 107-116.

[9] M. Edelstein, A remark on a theorem of M. A. Krasnoselski, Amer. Math. Monthly 73 (1966), 509-510.

[10] M. Edelstein and R. C. O'Brien, Nonexpansive mappings, asymptotic regularity and successive approximations, J. London Math. Soc. (2) 17 (1978), no. 3, 547-554.

[11] D. Göhde, Zum Prinzip der kontraktiven Abbildung, Math. Nachr. 30 (1965), 251-258.

[12] J.-P. Gossez and E. Lami Dozo, Some geometric properties related to the fixed point theory for nonexpansive mappings, Pacific J. Math. 40 (1972), 565-573.

[13] B. Halpern, Fixed points of nonexpanding maps, Bull. Amer. Math. Soc. 73 (1967), 957-961.

[14] N. Hirano, K. Kido, and W. Takahashi, Nonexpansive retractions and nonlinear ergodic theorems in Banach spaces, Nonlinear Anal. 12 (1988), no. 11, 1269-1281.

[15] S. Ishikawa, Fixed points and iteration of a nonexpansive mapping in a Banach space, Proc. Amer. Math. Soc. 59 (1976), no. 1, 65-71. 
[16] J. L. Kelley, General Topology, D. Van Nostrand, New York, 1955.

[17] W. A. Kirk, A fixed point theorem for mappings which do not increase distances, Amer. Math. Monthly 72 (1965), 1004-1006.

[18] M. A. Krasnosel'skiŭ, Two remarks on the method of successive approximations, Uspehi Mat. Nauk (N.S.) 10 (1955), no. 1(63), 123-127 (Russian).

[19] E. Lami Dozo, Multivalued nonexpansive mappings and Opial's condition, Proc. Amer. Math. Soc. 38 (1973), 286-292.

[20] P. -K. Lin, K. K. Tan, and H. K. Xu, Demiclosedness principle and asymptotic behavior for asymptotically nonexpansive mappings, Nonlinear Anal. 24 (1995), no. 6, 929-946.

[21] Z. Opial, Weak convergence of the sequence of successive approximations for nonexpansive mappings, Bull. Amer. Math. Soc. 73 (1967), 591-597.

[22] S. Prus, Banach spaces with the uniform Opial property, Nonlinear Anal. 18 (1992), no. 8, 697704.

[23] S. Reich, Weak convergence theorems for nonexpansive mappings in Banach spaces, J. Math. Anal. Appl. 67 (1979), no. 2, 274-276.

[24] G. Rodé, An ergodic theorem for semigroups of nonexpansive mappings in a Hilbert space, J. Math. Anal. Appl. 85 (1982), no. 1, 172-178.

[25] B. Sims, A support map characterization of the Opial conditions, Miniconference on Linear Analysis and Function Spaces (Canberra, 1984), Proc. Centre Math. Anal. Austral. Nat. Univ., vol. 9, The Australian National University, Canberra, 1985, pp. 259-264.

[26] T. Suzuki, Strong convergence theorem to common fixed points of two nonexpansive mappings in general Banach spaces, J. Nonlinear Convex Anal. 3 (2002), no. 3, 381-391.

[27] Common fixed points of two nonexpansive mappings in Banach spaces, Bull. Austral. Math. Soc. 69 (2004), no. 1, 1-18.

[28] T. Suzuki and W. Takahashi, Weak and strong convergence theorems for nonexpansive mappings in Banach spaces, Nonlinear Anal. 47 (2001), no. 4, 2805-2815.

[29] W. Takahashi, A nonlinear ergodic theorem for an amenable semigroup of nonexpansive mappings in a Hilbert space, Proc. Amer. Math. Soc. 81 (1981), no. 2, 253-256.

[30] R. Wittmann, Approximation of fixed points of nonexpansive mappings, Arch. Math. (Basel) 58 (1992), no. 5, 486-491.

[31] D. van Dulst, Equivalent norms and the fixed point property for nonexpansive mappings, J. London Math. Soc. (2) 25 (1982), no. 1, 139-144.

Tomonari Suzuki: Department of Mathematics, Kyushu Institute of Technology, Sensui-cho, Tobata-ku, Kitakyushu 804-8550, Japan

E-mail address: suzuki-t@mns.kyutech.ac.jp 


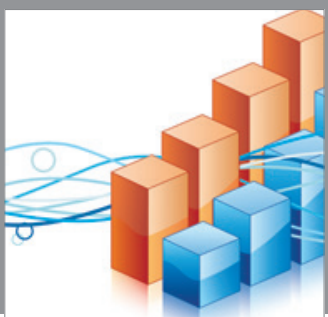

Advances in

Operations Research

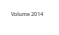

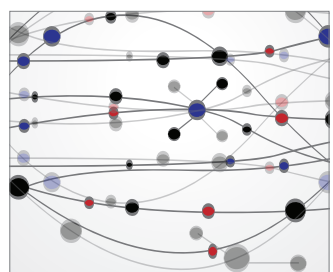

\section{The Scientific} World Journal
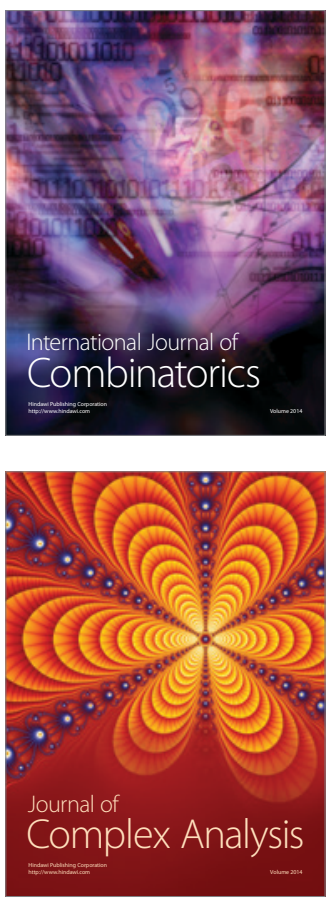

International Journal of

Mathematics and

Mathematical

Sciences
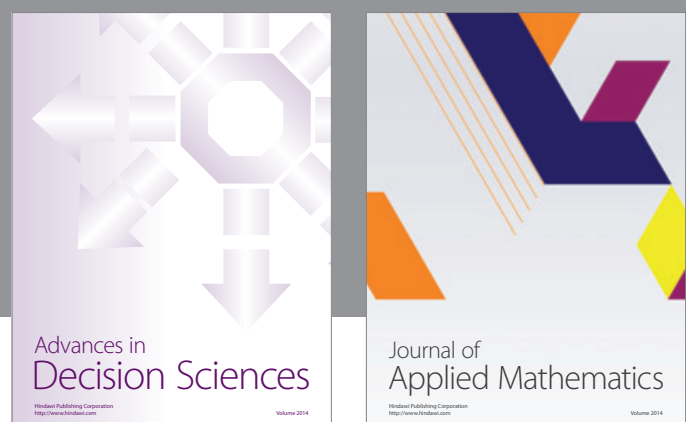

Journal of

Applied Mathematics
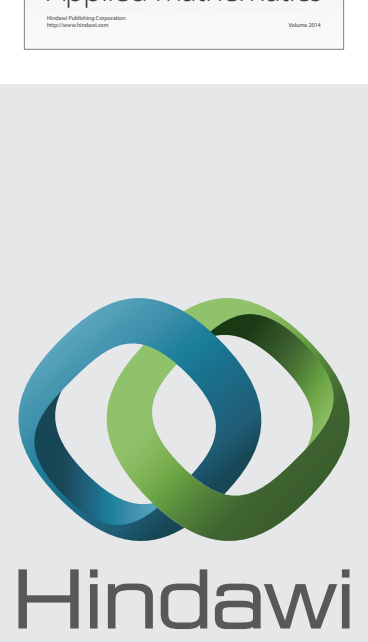

Submit your manuscripts at http://www.hindawi.com
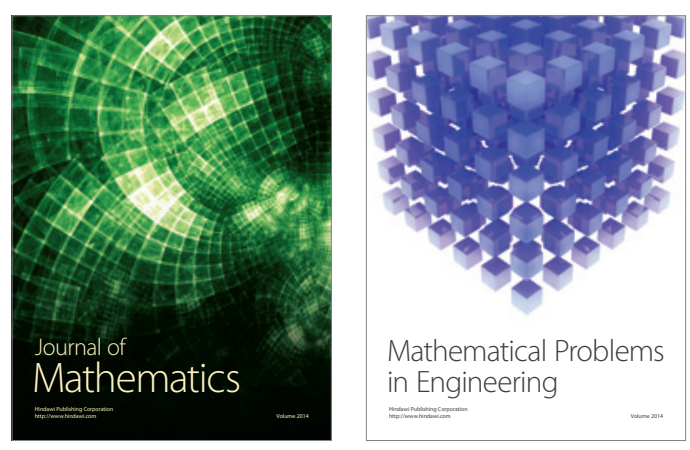

Mathematical Problems in Engineering
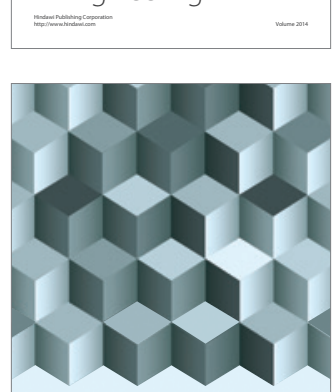

Journal of

Function Spaces
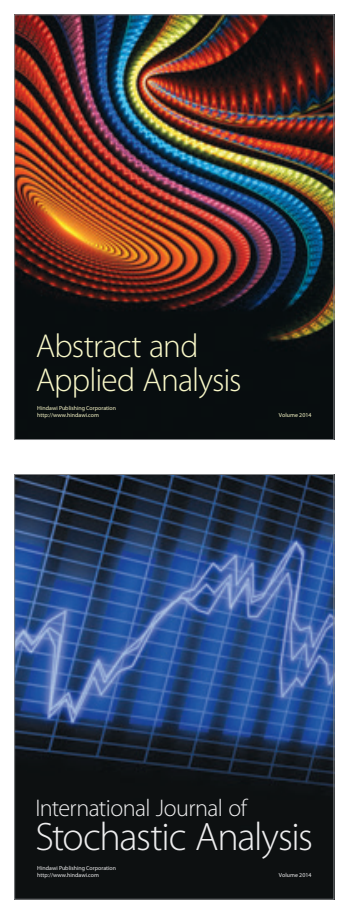

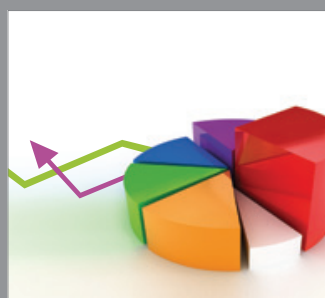

ournal of

Probability and Statistics

Promensencen
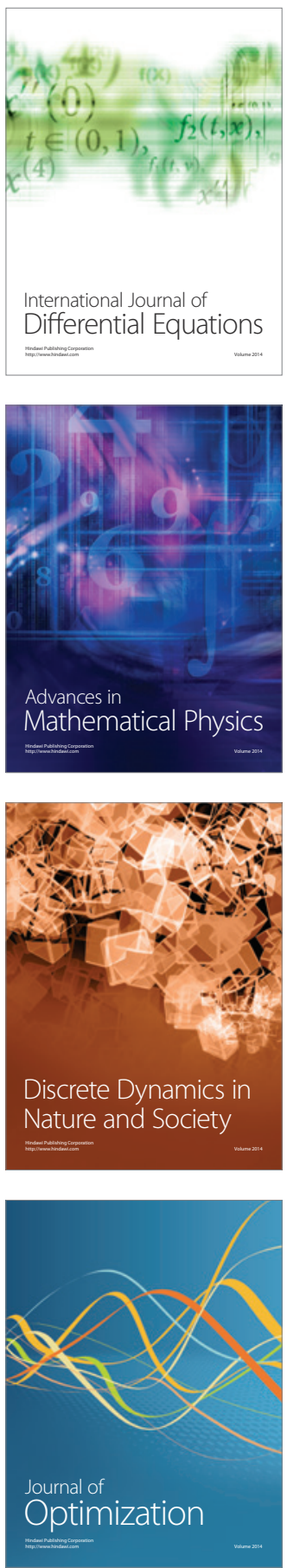